\title{
Cloning and Expression Analysis of a Squalene Epoxidase Gene from Ginkgo biloba
}

\section{Li ZHU, Liang-Qiong MA, Feng XU, Xiao-Juan YAN, Wei-Wei ZHANG*}

\author{
Yangtze University, College of Horticulture and Gardening, 434025,Jingzhou, China; Zhuli322@163.com; maliangqiong@163.com; \\ xufeng198@126.com;15335763736@163.com;wwzhangchn@163.com (*correspondingauthor)
}

\begin{abstract}
Squalene epoxidase is a key enzyme involved in triterpene saponins biosynthetic pathways in plants. In this study, the $S E$ gene was isolated from Ginkgo biloba by RT-PCR, which designated as GbSE, GenBank accession number: KY751713. The length of GbSE gene is $1646 \mathrm{bp}$, contains an open reading frame of 1617 bp encoding 538 amino acids. The theoretical molecular weight and pI of GbSE protein was $58.3 \mathrm{kDa}$ and 8.35 , respectively. Sequence multiple alignment found that GbSE protein had high homology with SE proteins from other plants, including several highly conservative motifs and amino acids. Phylogenetic tree analysis showed that Ginkgo biloba SE belonged to the gymnospermous group, and was closely related to the SE protein of Picea sitchensis. A study of gene expression analysis indicated that the GbSE gene was highly expressed in stems, low expressed in fruits. The cDNA sequence of GbSE gene was cloned from G. biloba and the expression level of this gene was analyzed. This work can provide some theoretical basis for the research on the molecular mechanism of triterpenoid saponin biosynthesis.
\end{abstract}

Keywords: cloning, expression profile, Ginkgo biloba, squalene epoxidase, triterpenoid saponin

\section{Introduction}

Ginkgo biloba, also named as Gongsun tree, Duck feet, belong to deciduous tree, is the only species of Ginkgo family, and is one of the oldest species in China (Zhou and Zheng, 2003). Due to $G$. biloba has survived from the fourth century of glacial coverage period so was known as "a living fossil” too (Xu et al., 2008). Extracts of G. biloba of active compounds is complex, not only contains flavonoids, including biflavone, flavonoid glycoside and flavonoid aglycones, which mainly play a role in eliminating free radical and anti-lipid peroxidation in plants, but also contains terpene lactones, including diterpenes and sesquiterpenes, they have a strong specific inhibitory activity on the receptor (van Beek, 2002; Cheng et al., 2009). Consequently, in the recent years, $G$. biloba especially as a medicinal plant has become one of popular functional plant favored by scholars, but mainly in the study of flavonoids little about triterpenoid saponin (Cao et al., 2012).

Triterpenoid saponin, when in vitro of plant, beneficial to human body as immune adjuvant and lower cholesterol, and in vivo of plant also defend plants against attack from external environment and enhance the ability of resistance to pests and diseases (Osbourn and Haralampidis, 2002;
Yendo et al., 2010). Triterpenoid saponins skeleton are synthesized by the isoprenoid pathway (Lambert et al., 2011). Oxidosqualene (also named as squalene-2,3epoxide), the precursor of the triterpenoid saponins in plants, is synthesized by mevalonate (MVA) pathway in the cytoplasm and 2-C-methyl-D-erythritol 4-phosphate (MEP) pathway in plastids. Both pathways can synthetic isopentenyl pyrophosphate (IPP), and then by the farnesyl diphosphate synthase (FPS) converted to farnesyl diphosphate (FPP), subsequently, catalyzed into squalene by squalene synthase (SS) (Kuzuyama, 2002; Vranová et al., 2013). Finally, under the effect of squalene epoxidase (SE) squalene is converted into oxidosqualene, which is the first oxygenation step in triterpenoid saponins biosynthesis (Fig. 1) (Haralampidis et al., 2002). Thus, squalene epoxidase is one of the rate-limiting and a key enzyme in the triterpenoid saponins biosynthetic pathway.

Squalene epoxidase, also known as squalene momooxygenases, which located in the endoplasmic reticulum of eukaryotic cell, with the flavin adenine dinucleotide (FAD) binding site. The FAD is involved in the triterpenoid saponins biosynthesis process of key enzymes important cofactor about redox reaction (Favre and Ryder, 1996). Thus far, squalene epoxidase has been isolated from different species, such as rat (Sakakibara et al., 1995), Candida albicans (Favre and Ryder, 1997), Panax ginseng (Han et al., 2010), Arabidopsis thaliana (Rasbery et 
40

al., 2007) and Panax notoginseng. Since SE is supposed to be a rate limiting enzyme, so its cloning and expression is found to play an important role in the biosynthesis of saponins. However, little is known about the SE involved in triterpenoid saponins biosynthesis in $G$. biloba. In the present study, we report for the first time the isolation, cloning, sequence analysis, tissue-specific expression of squalene epoxidase from $G$. biloba. We attempted to gain greater understanding of the regulatory role of squalene epoxidase in $G$. biloba triterpenoid biosynthesis. The results of this study may provide some theoretical reference and lay some molecular basis for the study of metabolic regulation of triterpenoid saponins and sterols biosynthesis.

\section{Materials and Methods}

\section{Plantmaterial}

$G$. biloba plants were grown in the Yangtze University, Jingzhou, China. The plants were used as source plant material for the present study. Six different tissues (roots, stems, leaves, male flowers, female flowers and fruits) that were used for RNA extraction and expression analysis were collected, fresh tissue samples were then immediately frozen in liquid nitrogen and stored at $-80{ }^{\circ} \mathrm{C}$ until further analysis.

\section{Total RNA extraction}

Different tissues of ginkgo were immediately pulverized in liquid nitrogen, total RNA was extracted from roots, stems, leaves, male flowers, female flowers and fruits following the instruction of TaKaRa MiniBEST Universal RNA Extraction Kit (TaKaRa, Dalian, China). The quality, concentration, and purity total RNA was detected at OD260/280 absorbance ratio by using a spectrophotometer and $1 \%(\mathrm{w} / \mathrm{v})$ agarose gel electrophoresis.

\section{The cDNA synthesis and cloning of GbSE}

First-strand cDNA was synthesized by using PrimeScript ${ }^{\mathrm{TM}}$ 1st Strand cDNA Synthesis Kit (TaKaRa, Dalian, China). According to the transcriptome sequencing data of $G$. biloba, the specific primer pair SE-FP (5'AGGTTAAGCCAGTGGCGACCTC-3') and SE-RP (5’- CTAATTATCTACTGAGGAAGCTGAATT-3') were designed and synthesized. The PCR reaction mixture was $\mathrm{ddH}_{2} \mathrm{O} 40.5 \mu \mathrm{L}, 10 \times$ Buffer $\left(\mathrm{Mg}^{2+}\right) 5 \mu \mathrm{L}$, dNTP (10 $\left.\mathrm{mmol} \cdot \mathrm{L}^{-1}\right) 1 \mu \mathrm{L}, \mathrm{SE}-\mathrm{FP}$ and SE-RP $1 \mu \mathrm{L}$, cDNA template 1 $\mu \mathrm{L}$, Taq DNA polymerase $\left(5 \mathrm{U} \cdot \mu \mathrm{L}^{-1}\right) 0.5 \mu \mathrm{L}$. The PCR reaction condition was followed as $94{ }^{\circ} \mathrm{C}$ for $5 \mathrm{~min}$; 30 cycles at $94^{\circ} \mathrm{C}$ for $30 \mathrm{~s}, 60^{\circ} \mathrm{C}$ for $30 \mathrm{~s}, 72^{\circ} \mathrm{C}$ for $1 \mathrm{~min}$; with an extension at $72{ }^{\circ} \mathrm{C}$ for $10 \mathrm{~min}$. The PCR amplification products were analyzed by $1 \%$ gel electrophoresis, and then the target gene was recovered by TaKaRa MiniBEST Agarose Gel DNA Extraction Kit (TaKaRa, Dalian, China). After further purification, it was cloned into pMD19-T vector (TaKaRa, Dalian, China), and transformed into Escherichia coli DH5a strain. The transformed bacteria were evenly applied to the plate for blue-white selection; the positive clones were validated by colony PCR and then sequenced in the Shanghai Sangon Biotechnology Company.

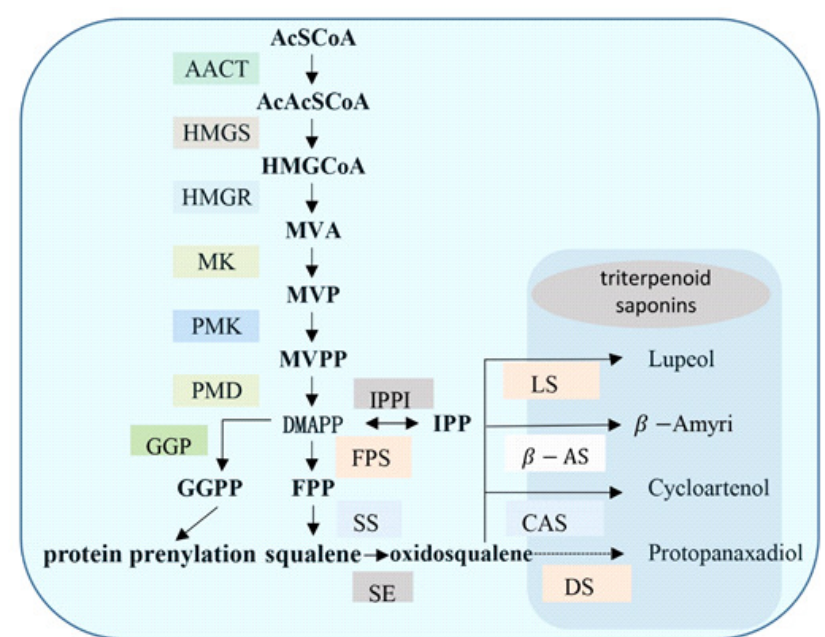

Fig. 1. The triterpenoid saponins biosynthesis of MVA pathway in G. biloba. AACT, Acetyl-CoA C-acetyltransferase; HMGS, 3-Hydroxy-3-methylglutaryl-CoA synthase; HMGR, 3-Hydroxy-3-methylglutaryl-CoA reductase; MK, MVA kinase; PMK, Phospho-MVA kinase; PMD, Diphospho-MVA decarboxylase; GGP, Geranyl diphosphate synthase; IPPI, Isopentenyl diphosphate; FPS, Farnesyl diphosphate synthase; SS, Squalene synthase; SE, Squalene epoxidase; LS, Lanosterol synthase; CAS, Cycloartenol synthase; DS, Dammarenediol synthase; $\beta$-AS, $\beta$-amyrin synthase

\section{Bioinformatics analyses}

The isolated nucleotide sequence and deduced amino acid sequence were compared through database search using online bioinformatics tools (NCBI, http://www.ncbi .nlm.nih.gov/BLAST/). Using Vector NTI Suite V 11.5 and DNAMAN 8 to analyze cDNA sequence of $G b S E$ gene. The online tool ExPASy (http://web.expasy.org /protparam/) was used to estimate the physicochemical properties of amino acid sequence of the GbSE. Program Align X (Vector NTI Suite V 11.5) was used to perform multiple alignment analysis of SE proteins from various plants, the Clustal X 2.0 and MEGA 6.0 were used to construct the phylogenetic tree of SE proteins with the neighbor-joining (NJ) method.

\section{Quantitative real-time PCR ( $q R T-P C R)$ analysis}

Total RNA was isolated from six different samples by using TaKaRa MiniBEST Universal RNA Extraction Kit. First-strand cDNA was synthesized using a PrimeScript ${ }^{\text {TM }}$ RT reagent Kit with gDNA Eraser (TaKaRa, Dalian, China). Real-time quantitative PCR were performed using the Mini OpticonTM Real-Time PCR system (Bio-Rad) with SYBR ${ }^{\odot}$ Premix Ex Taq ${ }^{\mathrm{TM}}$ II Kit (TaKaRa, Dalian, China). Relative mRNA levels were normalized to a housekeeping gene $(G A P D H)$ from ginkgo. The primer sequences of reference gene (GAPDH-F: 5'CTGGCGTAGAGTATGTGGTTGAAT-3', GAPDHR: 5'- CACGCCAACAACGAACATG-3') and GbSE (SE-RTF: 5'-CGACCGTTGAGTTTGGTTCTG-3', SERTR: (5'- TCTGTCTGACACCTTCTGCCTTA-3') for the qRT-PCR were synthesized by Shanghai Sangon Biotechnology Company. qRT-PCR reactions were 
performed in a final reaction volume of $25 \mu \mathrm{l}$ according to the manufacturer's protocol. Each reaction sample was prepared in three technical replicates with a negative control using water as template. The relative expression level of $G b S E$ gene was calculated by using the $2^{-\Delta \Delta C T}$ method (Livak and Schmittgen, 2001).

\section{Results}

\section{Cloning of GbSE cDNA sequence}

By PCR amplification, a cDNA sequence of SE was obtained from $G$. biloba, which was designated as $G b S E$ (GenBank accession no. KY751713). The cDNA sequence was $1646 \mathrm{bp}$, contains an open reading frame of $1617 \mathrm{bp}$, and encodes 538 amino acids (Fig. 2).

\section{Characterization of the deduced GbSE protein}

Putative GbSE protein contains 538 amino acids. The result of computer $\mathrm{pI} / \mathrm{Mw}$ analysis showed the molecular weight and isoelectric point of GbSE protein were $58.3 \mathrm{kD}$ and 8.35 , respectively. The secondary structure of $G b S E$ via SOPMA tool analysis showed that the percentages of alpha helix, extended strand, random coil and beta turn were $34.57 \%, \quad 23.79 \%, \quad 32.16 \%$ and $9.48 \%$, respectively. Homology analysis was completed with online tool BLASTP (NCBI) and Align X (Vector NTI 11.5). The results showed that GbSE protein belongs to NADB Rossmann superfamily, which contains the conservative domain structure FAD binding sites. Conservative areas are in red box, including G, L, RMR, RHPLTGGGMTV, and PDRIVGELLQPGG (Fig. 3). G and L are conservative sites that affect the function and associated with the active center of the enzyme. RxR conserved area plays an important role in the complexation of diphosphate after separation from the substrate (Starks and Noel, 1997). Domain motifs of the RHPLTGGGMTV and PDRIxGExxQPGG are the predicted FAD binding site (Sakakibara et al., 1995; Favre and Ryder, 1997). The putative GbSE protein sequence showed high identical to SE proteins from other plants (Fig. 3). The similarity between GbSE and SE proteins from Picea sitchensis (ABK24903), Amborella trichopoda

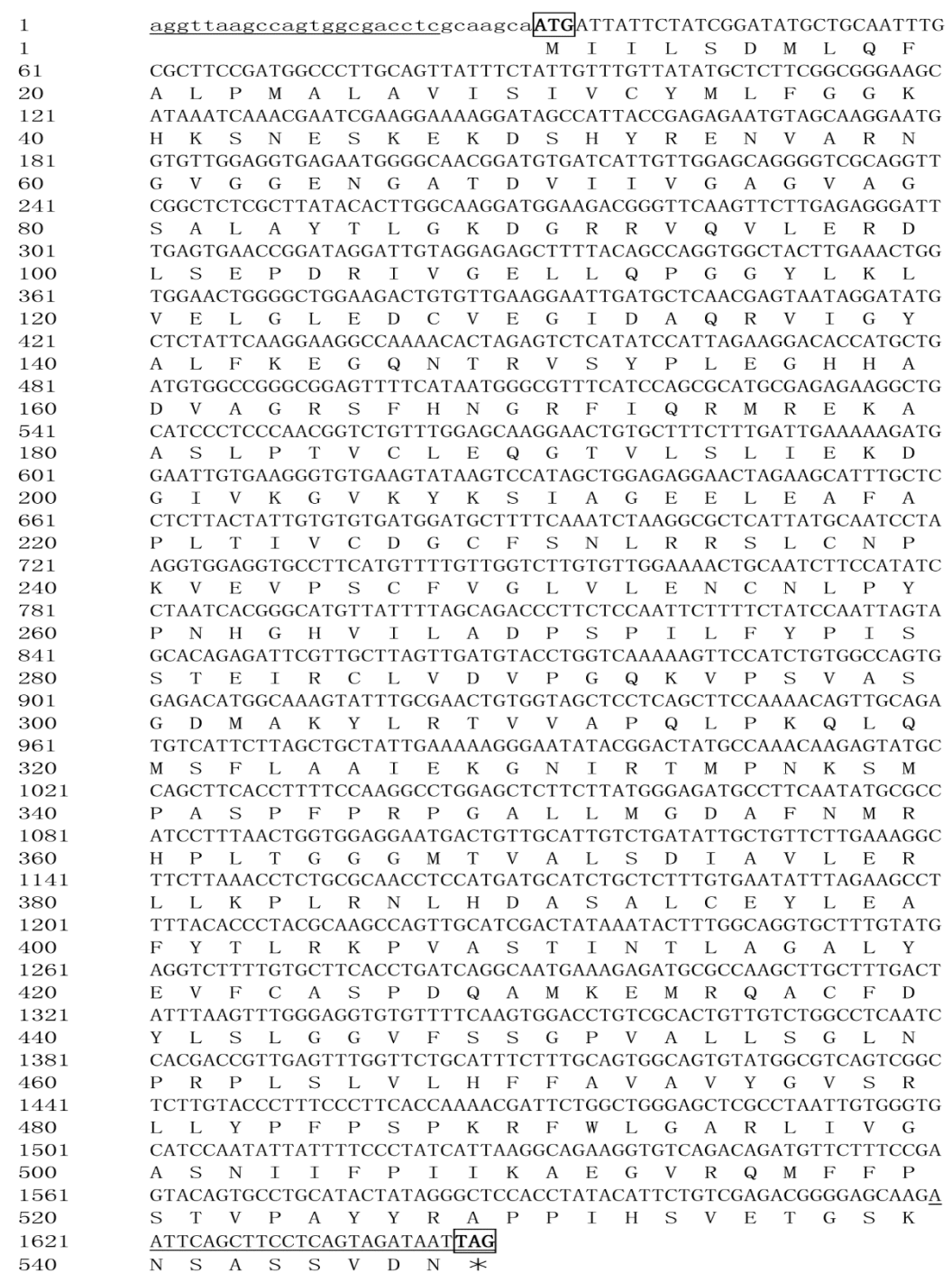

Fig. 2. The cDNA and deduced amino acid sequence of GbSE. The initial codon and the stop codon are highlighted in box, letters with underlined indicate the primer sequence 


\section{Ginkgo biloba \\ Vitis vinifera \\ Zostera marina \\ Picea sitchensis \\ Ananas comosus \\ Theobroma cacao \\ Manihot esculenta \\ Chlorophytum borivilianum}

Ginkgo biloba

Vitis vinifera

Zostera marina

Picea sitchensis

Ananas comosus

Theobroma cacao

Manihot esculenta

Chlorophytum borivilianum

Ginkgo biloba

Vitis vinifera

Zostera marina

Picea sitchensis

Ananas comosus

Theobroma cacao

Manihot esculenta

Chlorophytum borivilianum

Ginkgo biloba

Vitis vinifera

Zostera marina

Picea sitchensis

Ananas comosus

Theobroma cacao

Manihot esculenta

Chlorophytum borivilianum

Ginkgo biloba

Vitis vinifera

Zostera marina

Picea sitchensis

Ananas comosus

Theobroma cacao

Manihot esculenta

Chlorophytum borivilianum

Ginkgo biloba

Vitis vinifera

Zostera marina

Picea sitchensis

Ananas comosus

Theobroma cacao

Manihot esculenta

Chlorophytum borivilianum

Ginkgo biloba
Vitis vinifera
Zostera marina
Picea sitchensis
Ananas comosus
Theobroma cacao
Manihot esculenta
Chlorophytum borivilianum

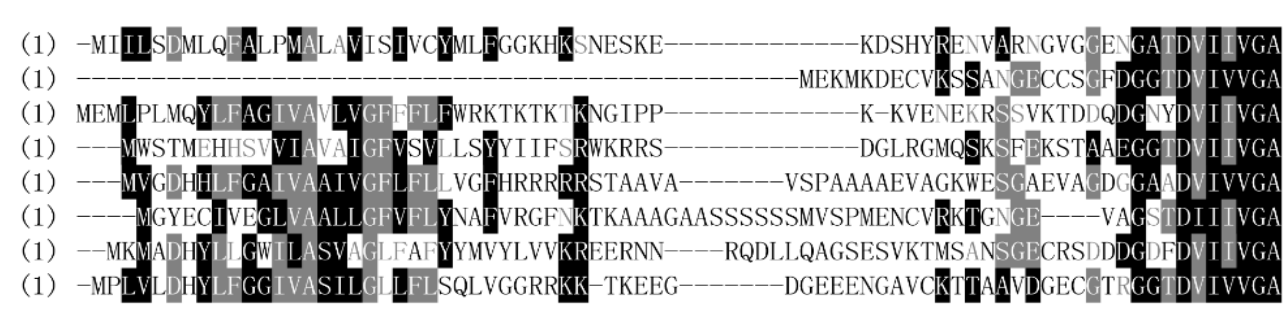

(67) GVAGSALAVTL GKDGRRVQVLERDL SIPDRIVGELLQPGGYLKLVELGL DCVEGIDAQRV IGYALFKEGQNIRVSYPLF (33) GVAGSAL AHTL GKDGRRVHVTERDLSGPDRIVGELLQPGGYLKLIELGLEDCL NEAQRV LGYALYKDGKNARLSYPLE (67) GVAGSALAYTL GKDGRRVRVIERDLSEPDRIVGELLQPGGYLKLVELDLEDCVEE IDAQRVNGYALFKDGR VTKVAYPLE (65) GVAGSALAYTLGKDGRRI I I ERDLSE PDRIVGELLQPGGYLKLIELGLDCVEGIDAQR IMGYALFKEGKDTK VYPLE (71) GVAGSAI AVTL GKDGRRVHVI ERDLSI PDRIVGELLQPGGYLKLI EL GLEDCVKI IDAQRVLGYALFKDGKNAKVSYPLE (73) GVAGSAL AYT F GKDGRRVHVI IERDLSF PDRIVGELLQPGGYLKL IEL GLEDCVDDIDAQQVF GYALYKDGKNT RLSYPLE (75) GVAGSAL AHTLGKDGRRVHVIERDLTE PDRIVGELLQPGGYLKL IELGLEDCMEEIDAQRV GYALFKDGKHTQLAYPLE (72) GVAGSALAYYTLGDGRRVHVIERDLTE PDRIVGELLQPGGYLLI ELGLQDCVGEIDAQRVLGYALFKDGRDTKVYPLE

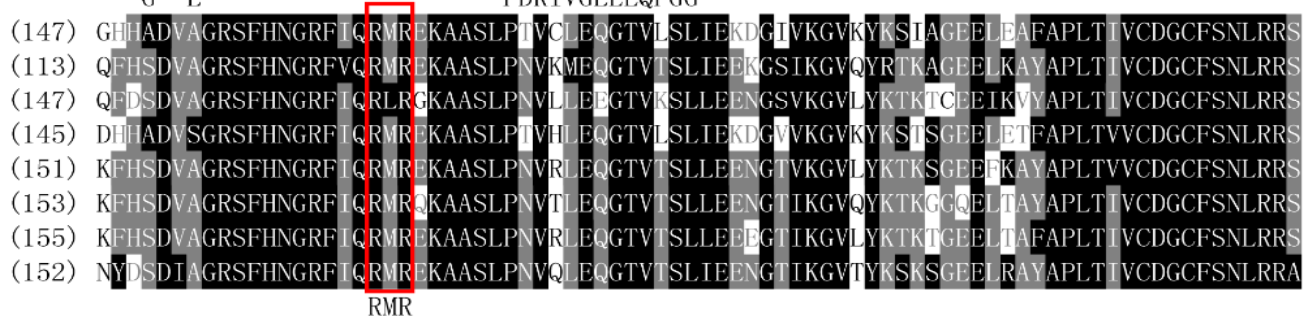

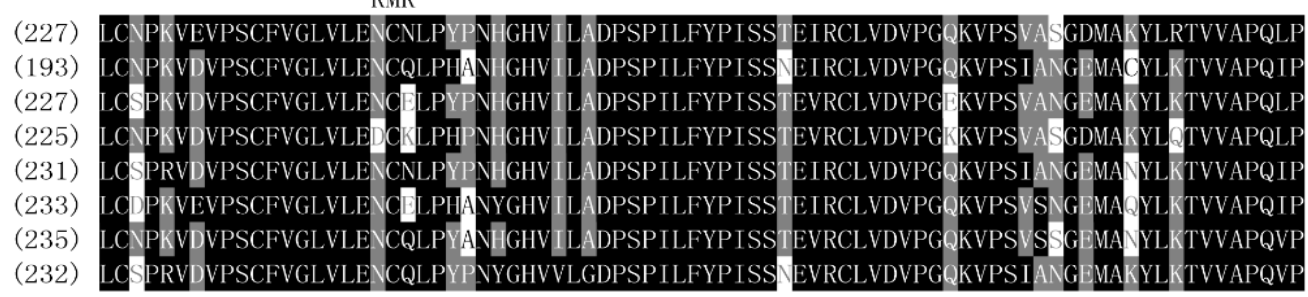
(232) LOSPRVDVPSCFVGLVLE VCQLPYPVYGHVVLGDPSPILFYPISS EVVCLVDVPGOKVPSI ANGEMAKYLKTVVAPQV
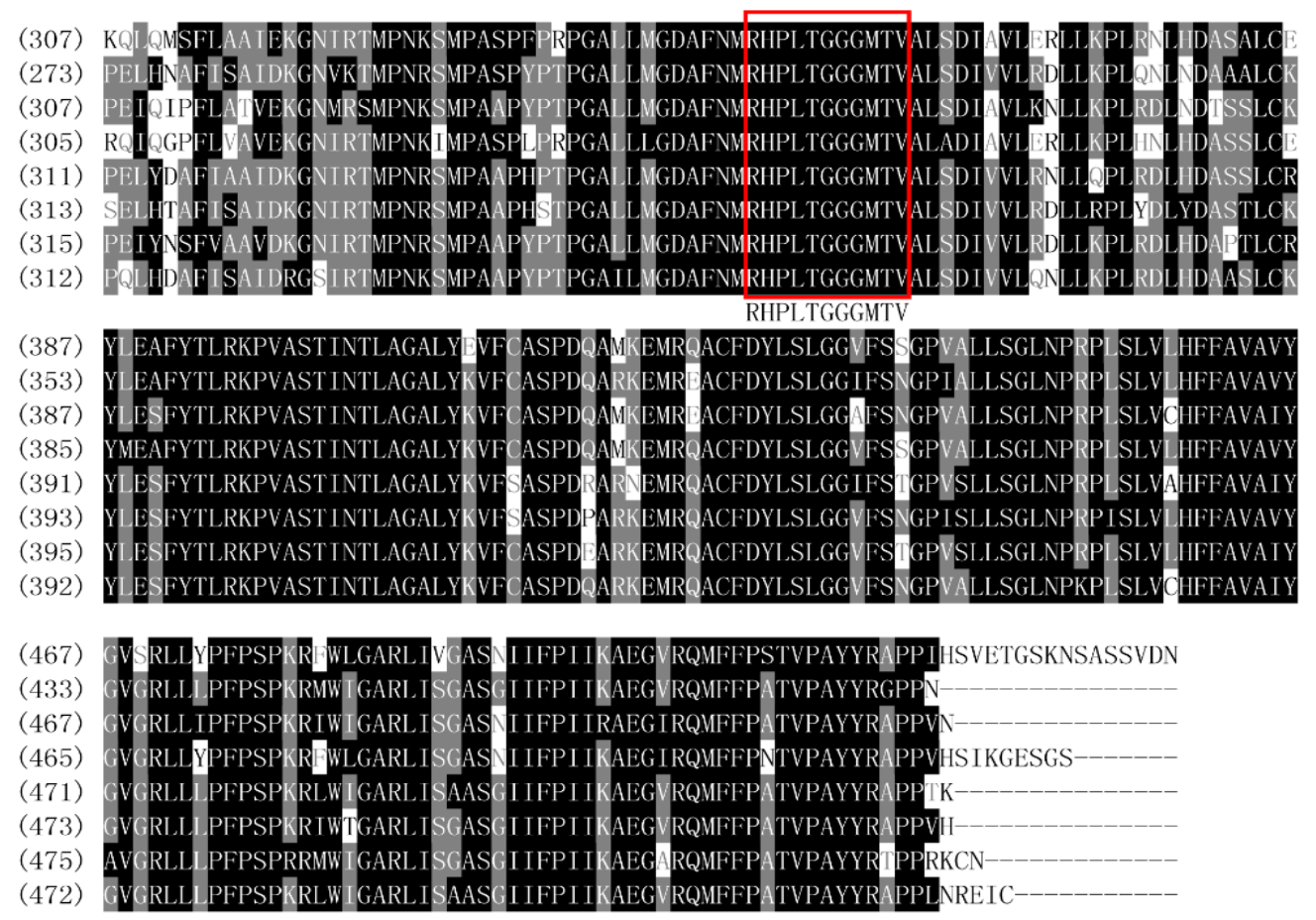

Fig. 3. Multiple alignment of amino acid sequences of SE. The completely identical amino acids are indicated with white foreground and black background. The conserved aminoacids are indicated with white foreground and grey background. Nonsimilar amino acids are indicated with black foreground and white background 
(XP_006857043), Zostera marina (KMZ75443), Ananas comosus (OAY63657), Chlorophytum borivilianum (AFN61200), Vitis vinifera (CBI25076), Zea mays (ONL95395), Manihot esculenta (OAY51053), Theobroma cacao (EOX97273) were $89 \%, 81 \%, 77 \%, 83 \%, 78 \%, 80 \%$, $81 \%, 83 \%, 81 \%$, respectively. The homologous sequence of $\mathrm{SE}$ among different species indicated the SE proteins might keep a conservative during the molecular evolution.

\section{Phylogenetic analysis of GbSE protein}

The phylogenetic tree was constructed by ClustalX 2.0 and MEGA 6.0 to further explore the evolutionary relationship between GbSE from other proteins involved in triterpenoid saponins biosynthesis in plants. Phylogenetic analysis indicated that all of SE proteins descended from a common progenitor. In addition, SE proteins from different plants were clearly clustered into three branches, gymnospermous, dicots and monocots of angiospermous (Fig. 4). As shown in the phylogenetic tree, SE of G. biloba and Picea sitchensis (ABK24903) were clustered into the
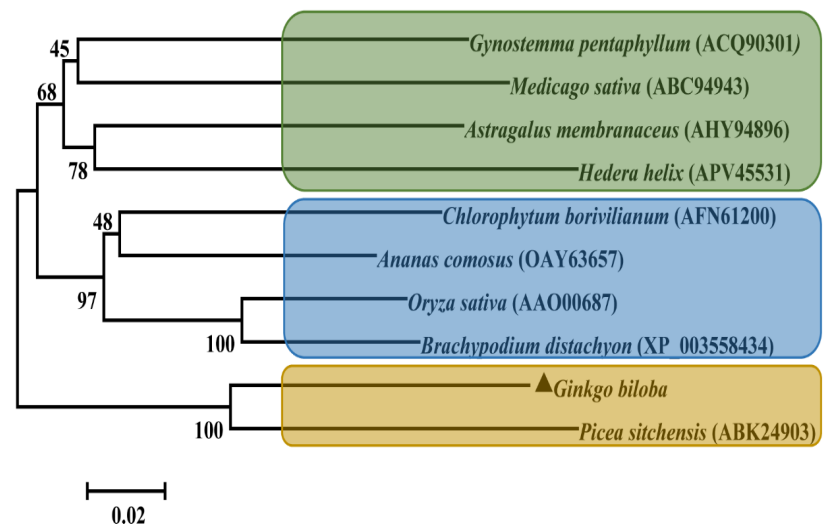

Fig. 4. Phylogenetic tree of SE from different plant species. The numbers at each node represented the bootstrap values (with 1000 replicates)

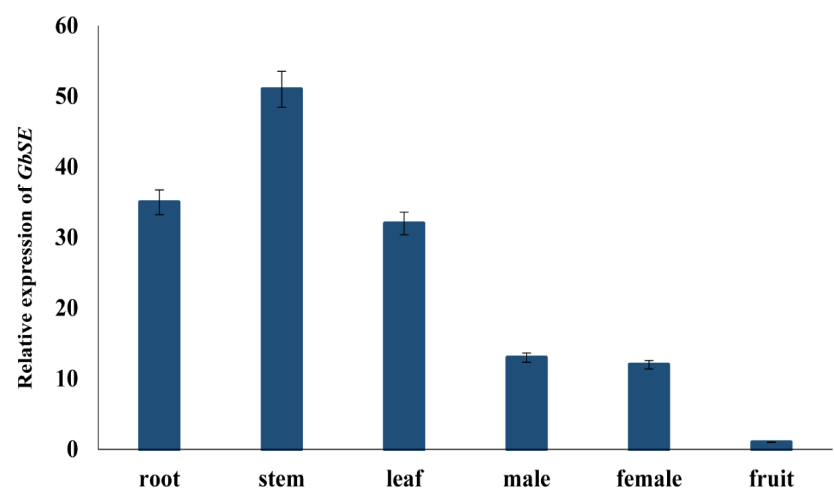

Fig. 5. Expression pattern of GbSE gene among different tissues of G. biloba

gymnospermous branch, suggesting they have the closer evolutionary distance. SE proteins from Ananas comosus (OAY63657), Oryza sativa (AAO00687), Brachypodium distachyon (XP_003558434), Chlorophytum borivilianum (AFN61200) were clustered into monocots branch of angiospermous, while SE proteins from Gynostemma pentaphyllum (ACQ90301), Hedera helix (APV45531), Astragalus membranaceus (AHY94896), Medicago sativa (ABC94943) were clustered into dicots branch of angiospermous. These results suggest that $\mathrm{SE}$ genes from the same plant family will be priority clustered together, followed by clustering with the adjacent plant families, which indicated that the $S E$ gene is conserved in the process of evolution, and this is consistent with the natural evolution of plants.

\section{Expression profile of the GbSE in different tissues}

Relative expression levels of $G b S E$ in different tissues of G. biloba was analyzed by Real-time PCR with specific primers. Total RNA was extracted from roots, stems, leaves, male flowers, female flowers and fruits. The results revealed that $G b S E$ was expressed in various tissues, but it was highly expressed in stems. Compared with GbSE mRNA levels in stems, GbSE mRNA levels showed significantly decrease in roots and leaves. The expression levels of GbSE in flowers were low, and no significant difference between male flowers and female flowers. The lowest expression level was observed in fruits (Fig. 5). The expression levels of GbSE in different tissues may be related to its function in G.biloba.

\section{Discussion}

Squalene epoxidase play important roles in triterpenoid saponins biosynthesis. In this study, the $S E$ gene was isolated from $G$. biloba, sequence analysis showed that it contains an open reading frame of $1617 \mathrm{bp}$, encodes 538 amino acids, the theoretical molecular weight and $\mathrm{pI}$ of GbSE protein were $58.3 \mathrm{kD}$ and 8.35 , respectively. Multiple alignment results showed that GbSE protein has high homologous with SE proteins from Picea sitchensis, Amborella trichopoda, Zostera marina, Ananas comosus, Chlorophytum borivilianum, Vitis vinifera, Zea mays, Manihot esculenta, Theobroma cacao. Phylogenetic tree analysis showed that SE proteins of Picea sitchensis and G. biloba from gymnospermous were clustered into the same branch, which indicated that $G b S E$ was belonging to $S E$ gene family. GbSE contains the FAD conservative domain structure, which bind to key enzymes as important cofactor of redox reaction in the biosynthesis process of triterpenoid saponins. In addition, $\mathrm{RxR}$ conserved is also found in other enzymes such as sesquiterpene synthase in Chamaemelum, plays an important role in the complexation of diphosphate after separation from the substrate (Cao et al., 2012).

Quantitative Real-time PCR analysis showed that GbSE display distinct tissue specific expression, it was higher expressed in the stems, lower expressed in the fruits. This phenomenon is probably related to the function of SE in $G$. biloba, Squalene epoxidase maybe also involved in triterpenoid saponins biosynthesis in G. biloba. Moreover, triterpenoid saponins can act as a chemical defense substance resistant to pests and diseases in plants (Mahato $e t$ al., 1988). In Ziziphus jujuba, triterpenoid saponin not only has the ability to scavenge free radicals and antioxidant, but also can be moisturizers and antibacterial for plants (Sun et al., 2012). Hence, the expression levels of GbSE in roots was high may also be related to the response of external 
44

biological stress. In the future, we will study the relationship between $G b S E$ expression, triterpenoid saponins content, and transcription factors. This research enables to improve understanding of key enzymes involved in triterpenoid saponins biosynthesis pathway.

\section{Acknowledgements}

This study was supported by the National Natural Science Foundation of China (31370680).

\section{References}

CaoF, ChengH, ChengS, LiL, XuF, Yu W, Yuan H(2012). Expression of selected Ginkgo biloba heat shock protein genes after cold treatment could be induced by other abiotic stress. International Journal of Molecular Sciences 13(5):5768-5788.

Cheng SY, Xu F, Wang Y (2009). Advances in the study of flavonoids in Ginkgo biloba leaves. Journal of Medicinal Plant Research 3(13):12481252.

Favre B, Ryder NS (1996). Characterization of squalene epoxidase activity from the dermatophyte Trichophyton rubrum and its inhibition by terbinafine and other antimycotic agents. Antimicrobial Agents and Chemotherapy 40(2):443-447.

Favre B, Ryder NS (1997). Cloning and expression of squalene epoxidase from the pathogenicyeast Candida albicans. Gene 189(1):119-126.

Gawel NJ,Jarret RL (1991). A modified CTABDNA extraction procedure for Musa and Ipomoea. Plant Molecular Biology Reporter 9(3):262-266.

Han JY, Jungyo I, Yongsoo K, Yongeui C (2010). Regulation of ginsenoside and phytosterol biosynthesis by RNA interferences of squalene epoxidase gene in Panax ginseng. Phytochemistry 71(1):36-46.

Haralampidis K, Trojanowska M, Osbourn AE (2002). Biosynthesis of triterpenoid saponins in plants. Advances in Biochemical Engineering $75(75): 31-49$.

Kuzuyama T (2002). Mevalonate and nonmevalonate pathways for the biosynthesis of isoprene units. Bioscience Biotechnology and Biochemistry 66(8):1619-1627.

Lambert E, Faizal A, Geelen D (2011). Modulation of triterpene saponin production: in vitro cultures, elicitation, and metabolic engineering. Applied Biochemistry and Biotechnology 164(2):220-237.
Livak KJ, Schmittgen TD (2001). Analysis of relative gene expression data using Real-Time Quantitative PCR and the $2^{-\Delta \mathrm{C}^{\mathrm{T}}}$ Method. Methods 25(4):402-408.

Mahato SB, Sarkar SK, Poddar G (1988). Triterpenoid saponins. Phytochemistry 27(10):3037-3067.

Osbourn AE, Haralampidis K (2002). Chapter Five Triterpenoid saponin biosynthesis in plants. Recent Advances in Phytochemistry 36(02):8193.

Rasbery JM, Shan H, Leclair RJ, Norman M, Matsuda SP, Bartel B (2007). Arabidopsis thaliana squalene epoxidase 1 is essential for root and seed development. Journal of Biological Chemistry282(23):17002-17013.

Sakakibara J, Watanabe R, Kanai Y, Ono T (1995). Molecular cloning and expression of rat squalene epoxidase. Journal of Biological Chemistry 270(1):17-20.

Starks CM, Noel JP (1997). Structural basis for cyclic terpene biosynthesis by tobacco 5-epi-aristolochene synthase. Science 277(5333):1815-1820.

Sun YF, Liang ZS, Liu Z, Huang J, Yan H (2012). Study on antimicrobial and antioxidant activities of triterpenoid saponins in sour jujube fruits. Science and Technology of Food Industry 33(6):139-142.

van Beek TA (2002). Chemical analysis of Ginkgo biloba leaves and extracts. Journal of Chromatography A 967(1):21-55.

VranováE, Coman D, Gruissem W (2013). Network Analysis of the MVA and MEP Pathways for Isoprenoid Synthesis. Plant Biology $64(64): 463-$ 476.

Xu F, Cheng H, Cai R, Li LL, Chang J, Zhu J, Zhang XF, Chen LJ, Wang Y, Cheng SH, Cheng SY (2008). Molecular cloning and function analysis of an anthocyanidin synthase gene from Ginkgo biloba, and its expression in abiotic stress responses. Molecules and Cells 26(6):536547.

Yendo ACA, Costa FD, Gosmann G, Fettneto AG (2010). Production of Plant Bioactive Triterpenoid Saponins: Elicitation Strategies and Target Genes to Improve Yields. Molecular Biotechnology 46(1):94-104.

Zhou Z, Zheng S (2003). Palaeobiology: The missing link in Ginkgo evolution. Nature 423(6942):821-822. 